\title{
Testing Higgs sector of 2HDM
}

\section{Maria Krawczyk ${ }^{* i}$}

Institute of Theoretical Physics, Warsaw University, Poland

E-mail: Maria.Krawczyk@fuw.edu.pl

Properties of the Higgs sector of Two Higgs Doublet Model (2HDM) and existing constraints on its parameters are discussed. Potential of the Photon Linear Collider in testing various Higgs scenarios of 2HDM, including the MSSM, based on the realistic simulations is also presented.

International Europhysics Conference on High Energy Physics

July 21st - 27th 2005

Lisboa, Portugal

\footnotetext{
* Speaker.

${ }^{\dagger}$ Supported by the Polish Committee for Scientific Research, grant no. 1 P03B 04026 and project no. 115/E343/SPB/DESY/P-03/DWM517/2003-2005.
} 


\section{Symmetries of the 2HDM [1]}

The Higgs sector with two scalar complex doublets $(Y= \pm 1)$ is the simplest yet very rich extension of the Standard Model (SM). The general 2HDM potential is

$$
\begin{gathered}
V=\frac{1}{2} \lambda_{1}\left(\phi_{1}^{\dagger} \phi_{1}\right)^{2}+\frac{1}{2} \lambda_{2}\left(\phi_{2}^{\dagger} \phi_{2}\right)^{2}+\lambda_{3}\left(\phi_{1}^{\dagger} \phi_{1}\right)\left(\phi_{2}^{\dagger} \phi_{2}\right)+\lambda_{4}\left(\phi_{1}^{\dagger} \phi_{2}\right)\left(\phi_{2}^{\dagger} \phi_{1}\right)+\frac{1}{2}\left[\lambda_{5}\left(\phi_{1}^{\dagger} \phi_{2}\right)^{2}+\text { h.c. } .11 .1\right) \\
+\left\{\left[\lambda_{6}\left(\phi_{1}^{\dagger} \phi_{1}\right)+\lambda_{7}\left(\phi_{2}^{\dagger} \phi_{2}\right)\right]\left(\phi_{1}^{\dagger} \phi_{2}\right)+\text { h.c. }\right\}-\frac{1}{2}\left\{m_{11}^{2}\left(\phi_{1}^{\dagger} \phi_{1}\right)+\left[m_{12}^{2}\left(\phi_{1}^{\dagger} \phi_{2}\right)+\text { h.c. }\right]+m_{22}^{2}\left(\phi_{2}^{\dagger} \phi_{2}\right)\right\},
\end{gathered}
$$

with possible complex coefficients: $\lambda_{5}, \lambda_{6}, \lambda_{7}$, and $m_{12}^{2}$ (i.e. in total 14 real parameters). There is no $\left(\phi_{1}, \phi_{2}\right)$ transition (as a consequence there is no FCNC nor CPV) if $V$ is $Z_{2}$ symmetric under transformations: $\phi_{1} \rightarrow-\phi_{1}, \phi_{2} \rightarrow \phi_{2}$ (or vice versa). This means that $\lambda_{6}=\lambda_{7}=m_{12}^{2}=0$. Hard violation of $Z_{2}$ symmetry typically means appearance of quartic terms with $\lambda_{6}$, $\lambda_{7}$, while soft violation of $Z_{2}$ symmetry $\left(\lambda_{6}, \lambda_{7}=0\right)$ is governed by $m_{12}^{2}$.

Two fields with identical quantum numbers can mix without changing a physical picture. Such mixing is described by a global unitary transformation $\mathrm{U}(1) \mathrm{x} \mathrm{SU}(2)$ :

$$
\left(\begin{array}{c}
\phi_{1}^{\prime} \\
\phi_{2}^{\prime}
\end{array}\right)=e^{-i \rho_{0}}\left(\begin{array}{cc}
\cos \theta e^{i \rho / 2} & \sin \theta e^{i(\tau-\rho / 2)} \\
-\sin \theta e^{-i(\tau-\rho / 2)} & \cos \theta e^{-i \rho / 2}
\end{array}\right)\left(\begin{array}{c}
\phi_{1} \\
\phi_{2}
\end{array}\right) .
$$

It induces changes in the parameters of potential $V$ and the whole Lagrangian $L$.

Reparametrization transformations (RPaT): $\lambda_{i} \rightarrow \lambda_{i}^{\prime}$ and $m_{i j}^{2} \rightarrow\left(m^{\prime}\right)_{i j}^{2}$, constitute a 3 parametrical group with parameters: $\rho, \theta, \tau$ (here $\rho_{0}$ is not relevant) acting in the 14-dim space of Lagrangians. (The rephasing transformation group $(\theta=\tau=0)$ has one parameter only - $\rho$.) As various Lagrangians are reparametrization invariant there is a reparametrization equivalent 3-dim subspace of Lagrangians. Of course physical observables, like masses, are invariant under the RPaT. A CP violation in the Higgs sector is signalized by the complex parameters of the Lagrangian.

The U(1) $Q E D$ symmetric vacuum corresponds to the lowest energy than the "charged vacuum"; it can be choosen as

$$
\left\langle\phi_{1}\right\rangle=\frac{1}{\sqrt{2}}\left(\begin{array}{c}
0 \\
v_{1}
\end{array}\right),\left\langle\phi_{2}\right\rangle=\frac{1}{\sqrt{2}}\left(\begin{array}{c}
0 \\
v_{2} e^{i \xi}
\end{array}\right) .
$$

The rephasing of fields can always remove the phase difference $\xi \rightarrow \xi-\rho$. Instead of the oryginal parameters $m_{i j}^{2}$ from eq.(1) one can introduce $v_{1}, v_{2}$ (with $v_{1}^{2}+v_{2}^{2}=v^{2}, v=246 \mathrm{GeV}$ ) and $v=$ $\operatorname{Re} m_{12}^{2} / 2 v_{1} v_{2}$. Note, that the famous parameter $\tan \beta=v_{2} / v_{1}$ depends on reparametrization!

In $2 \mathrm{HDM}$ there are 5 physical Higgs bosons: three neutral ones $h_{1}, h_{2}, h_{3}$ and two charged $H^{ \pm}$. If there is no $\mathrm{CP}$ violation physical neutral particles $h, H, A$ have definite $\mathrm{CP}$ properties, otherwise $h_{1}, h_{2}, h_{3}$ are mixtures of $h, H, A$ states. Small or large mixing angles lead, respectively, to weak or strong $\mathrm{CP}$ violation (by mixing).

The Yukawa Lagrangian for quarks (similarly fo leptons) is

$$
-\mathscr{L}_{\mathrm{Y}}=\bar{Q}_{L}\left[\left(\Gamma_{1} \phi_{1}+\Gamma_{2} \phi_{2}\right) d_{R}+\left(\Delta_{1} \tilde{\phi}_{1}+\Delta_{2} \tilde{\phi}_{2}\right) u_{R}\right]+\text { h.c.. }
$$

$\Gamma_{1}=\Delta_{2}=0$ with diagonal real $\Gamma_{2}, \Delta_{1}$ corresponds to so called Model II (MSSM is of this type). 
We argue that $2 \mathrm{HDM}$ (II) with weak $\mathrm{CP}$ violation (small mixing angles), soft $Z_{2}$ violation and small $v$ is natural. This means that non-decoupling of heavy Higgs bosons, absent for large $v$, is a natural property of $2 \mathrm{HDM}$, in contrast to MSSM.

There are various relations between physical couplings of Higgs bosons to other particles, which can be used to test the model. It is useful to introduce relative couplings $\chi_{j}^{(i)}=g_{j}^{(i)} / g_{j}^{\mathrm{SM}}$ $(j=V, u, d)$, with sum rules $\Sigma_{i}\left(\chi_{j}^{(i)}\right)^{2}=1$. In addition there is a pattern relation, which hold for each neutral Higgs particle $h_{i}$ (in particular also for $h, H, A$ in the case of CP conservation):

$$
\left(\chi_{u}^{(i)}+\chi_{d}^{(i)}\right) \chi_{V}^{(i)}=1+\chi_{u}^{(i)} \chi_{d}^{(i)},\left(\chi_{u}^{(i)}-\chi_{V}^{(i)}\right)\left(\chi_{V}^{(i)}-\chi_{d}^{(i)}\right)=1-\left(\chi_{V}^{(i)}\right)^{2} .
$$

One can determine $\tan \beta=v_{2} / v_{1}=\tan \beta_{I I}$ via equations which hold in 2HDM (II) for $h_{i}$ and also for $h, H, A$ (except the last one, absent for $h, H$ ):

$$
\tan ^{2} \beta_{I I}=\frac{\left(\chi_{V}^{(i)}-\chi_{d}^{(i)}\right)^{*}}{\chi_{u}^{(i)}-\chi_{V}^{(i)}}=\frac{1-\left|\chi_{d}^{(i)}\right|^{2}}{\left|\chi_{u}^{(i)}\right|^{2}-1}=\frac{\operatorname{Im} \chi_{d}^{(i)}}{\operatorname{Im} \chi_{u}^{(i)}} .
$$

Neutral Higgs bosons couple to photons via loops with all charged particles existing in the theory. For the effective vertex $h_{i} \gamma \gamma$ the coupling $h_{i} H^{+} H^{-}$contributes:

$$
\chi_{H^{ \pm}}^{(i)}=\left(1-\frac{M_{i}^{2}}{2 M_{H^{ \pm}}^{2}}\right) \chi_{V}^{(i)}+\frac{M_{i}^{2}-v v^{2}}{2 M_{H^{ \pm}}^{2}} \operatorname{Re}\left(\chi_{u}^{(i)}+\chi_{d}^{(i)}\right) .
$$

For small $v$ and large $M_{H^{ \pm}}$even if all $\chi_{V}, \chi_{u}, \chi_{d}$ are equal 1 (SM-like scenario) $\chi_{H^{ \pm}}^{(i)} \approx 1$ there may occur large non-decoupling effects due to $H^{ \pm}$. Contrary, for large $v \sim M_{H^{ \pm}}^{2} / v^{2}$ one gets $\chi_{H^{ \pm}}^{(i)} \approx 0$.

\section{Constrainig 2HDM from the leptonic tau decays [2]}

The CP conserving $2 \mathrm{HDM}$ was tested at LEP in: Bjorken process $Z \rightarrow Z h$ (constraining $\chi_{V}^{(h)}=$ $\sin (\beta-\alpha))$, pair production $e^{+} e^{-} \rightarrow h A(\cos (\beta-\alpha))$, Yukawa process $e^{+} e^{-} \rightarrow b b h / A, \tau \tau h / A$ $\left(\chi_{d}^{(h / A)}\right)$. Also $e^{+} e^{-} \rightarrow H^{+} H^{-}$and a loop process $Z^{*} \rightarrow h / A \gamma$ were used to constrain various couplings and masses. Still, one very light neutral Higgs boson $h$ (with small $\chi_{V}$ ) or $A$ may exist with relatively large Yukawa coupling to $b, \tau$. Also precise $(g-2)_{\mu}$ data do not rule out such light Higgs scenario in 2HDM (e.g. for small $v$ and light $A$, with $\chi_{d} \in$ between 40-100). Note, that $H^{ \pm}$ has to be heavier than $490 \mathrm{GeV}$, as follows from the analysis of the process $b \rightarrow s \gamma$ [3].

New constraints of $2 \mathrm{HDM}$ were obtained from the leptonic tau decays using the ' 04 world data. In SM these decays proceed at the tree-level via $W^{ \pm}$exchange. In $2 \mathrm{HDM}$ charged-Higgsboson can be exchanged at tree-level, in addition. We found that 2HDM loop corrections, involving also neutral Higgs bosons, dominate at large $\tan \beta$, giving contribution to the branching ratios proportional to $\sim \tan \beta^{2}\left[\ln M_{h} / M_{H+}+1\right]$.

For the beyond SM contributions, defined as $B r^{l}=\left.B r^{l}\right|_{S M}\left(1+\Delta^{l}\right)\left[\left.B r^{l}\right|_{S M}=\left.\Gamma^{l}\right|_{S M} \tau_{\tau}^{\exp }\right]$, we derived the $95 \%$ C.L. bounds for the electron and muon decay modes:

$$
\left(-0.80 \leq \Delta^{e} \leq 1.21\right) \% \quad\left(-0.76 \leq \Delta^{\mu} \leq 1.27\right) \% .
$$

From this we got upper limits on Yukawa couplings for both light $h$ and light $A$ scenarios (Fig.1,left). New lower limit on mass of $M_{H^{ \pm}}$, which differs significantly from a standard constraint (based on the tree-level $H^{ \pm}$exchange only) was obtained (Fig. 1, right). We obtained also a upper limit on $M_{H^{ \pm}}$: e.g. for $\tan \beta=80, M_{A}=100$ and $M_{h}=20 \mathrm{GeV}$ mass of $H^{ \pm}$should be below $700 \mathrm{GeV}$. 

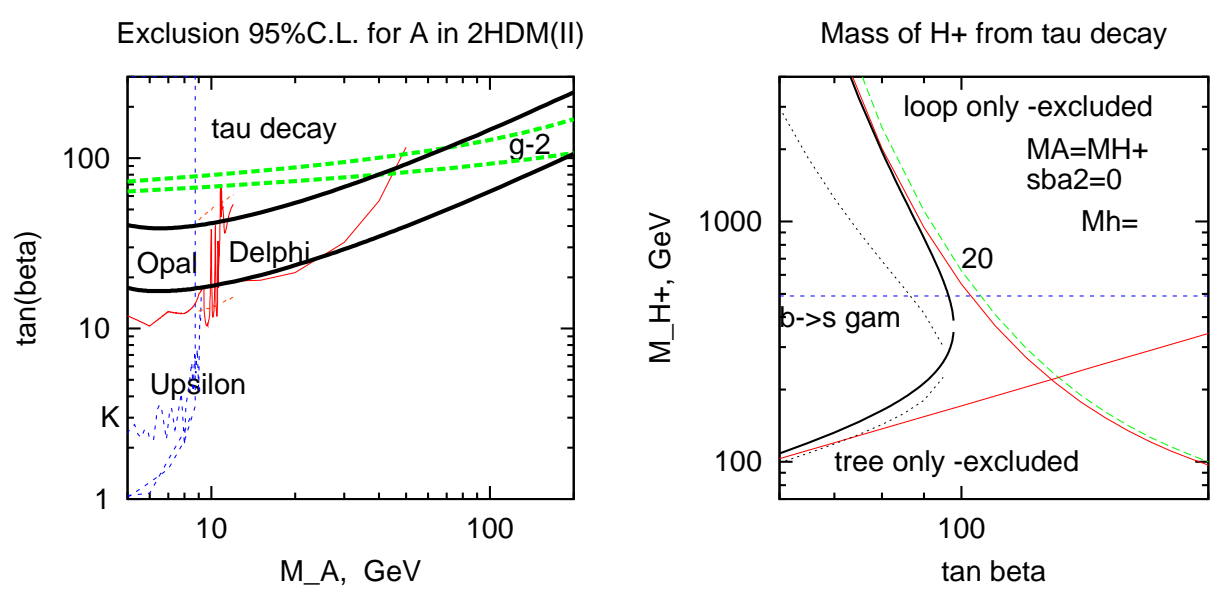

Figure 1: left: Constraints for $A$; right: Limits $M_{H^{ \pm}}$versus $\tan \beta$, dotted line for $M_{A}=100 \mathrm{GeV}$.

\section{Higgs-bosons production at PLC}

Higgs boson can be produced as a resonance at the Photon Linear Collider (PLC) in the collision of two photon beams obtained from the Compton backscattering processes. PLC is an ideal machine for testing properties of neutral Higgs bosons, especially CP parities. Energy and polarization spectrum of photons has to be incorporated in the realistic simulations.

The production relies on loop coupling $h_{i} \gamma \gamma$, mentioned above. For light Higgs boson the main decay channel is $b \bar{b}$, for heavier Higgses - the WW/ZZ. In MSSM the lightest Higgs boson has a property of that of SM, then, according to the sum rules, $H / A$ decay predominately to $b$ quarks.

\subsection{Covering the LHC wedge at PLC [4]}

The state-of-art analysis of $h \rightarrow b \bar{b}$ and $A, H \rightarrow b \bar{b}$ bases on realistic photon energy spectra (TESLA-like) and includes effects of the beams crossing angle and primary vertex distribution, as well as the realistic detector simulation (SIMDET). The NLO QCD background $Q \bar{Q}(g)(Q=c, b)$ as well as backgrounds due to $W^{+} W^{-}, q \bar{q}(q=u, d, s)$ are included. Overlaying events (OE; about 1 - 2 per bunch crossing) were taken into acount. Realistic b-tagging and corrections for escaping neutrinos were applied.

For the SM Higgs boson one obtains accuracy of the cross section measurements of the order $2-8 \%$ for $M_{h}=120-160 \mathrm{GeV}$ (with additional cuts for the $W W$ background).

The study for MSSM was performed to check potential of the PLC to cover the LHC wedge, where only one SM-like $h$ can be seen. Four MSSM parameter sets, for $\tan \beta=3-20$ and $M_{A}=$ $200-350 \mathrm{GeV}$ were considered. Results are shown in Fig. 2. (E.g. for set I precision is $11-21 \%$ for $\tan \beta=7$ after one year). For $M_{A}=300 \mathrm{GeV}$ one can discover MSSM Higgs bosons below $\tan \beta$ $=15$ (LHC limit) at PLC, and therefore there is a chance to cover LHC wedge.

\subsection{Determination of 2HDM couplings at PLC. Combined LHC, ILC and PLC analysis [5]}

Measurements at LHC, ILC and PLC are complementary, being sensitive to different Higgsboson couplings [5, 6]. This is shown in Fig. 3, left, where the ratios of the cross sections times BR 

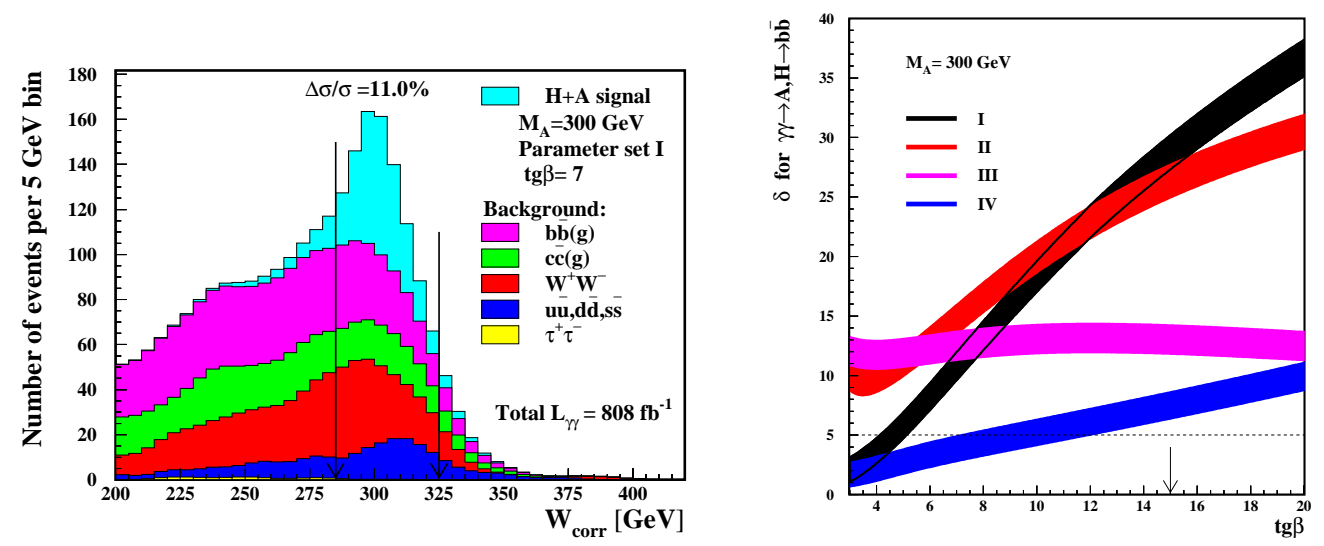

Figure 2: left: Corrected inv. mass for $M_{A}=300 \mathrm{GeV}$;right:Statistical signifi cance as a function of $\tan \beta$.
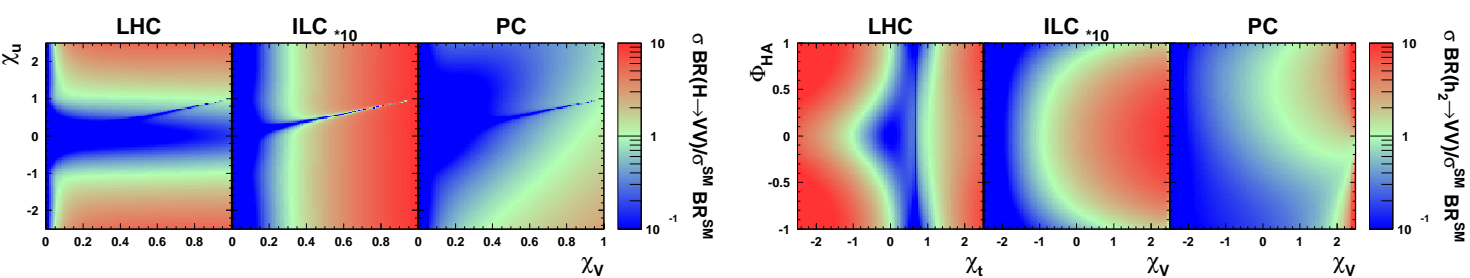

Figure 3: Expected production rates for CP conserving (violating) 2HDM: left(right).

to WW, obtained in the CP conserving 2HDM and SM, are presented as functions of $\chi_{V}^{H}$ and $\chi_{u}^{H}$ for $M_{H}=250 \mathrm{GeV}\left(M_{h}=120 \mathrm{GeV}, M_{H^{+}}=800 \mathrm{GeV}\right)$. Results for $2 \mathrm{HDM}(\mathrm{II})$ with weak CP violation via H-A mixing show similar compementarity (Fig.3, right). Here measurements of mixing phase $\phi_{H A}$ and the $\chi_{u}^{(2)}$ for LHC, and $\chi_{V}^{(2)}$ at ILC and PLC, are presented for $h_{2}$ with mass $250 \mathrm{GeV}$.

Simultaneous fit to the invariant mass distribution for $W^{+} W^{-} / Z Z$ at LHC, ILC and PC was performed with 12 parameters: $\chi_{V}, \chi_{u}, M_{H}, \phi_{H A}$ and 8 parameters describing normalization and PLC luminosity spectra shape, corresponding to following systematic uncertainties: signal normalization at LHC (ILC,PLC): 20\%(5,5), background normalization at LHC (ILC,PLC): 10\%(5,10), and PLC spectra shape: $10 \%$.

In general case the combined analysis of LHC, ILC and PLC data is needed.

\section{References}

[1] I. F. Ginzburg and M. Krawczyk, arXiv:hep-ph/0408011, Phys. Rev. D to appear

[2] M. Krawczyk and D. Temes, Eur. Phys. J. C 44, 435 (2005) [arXiv:hep-ph/0410248].

[3] P. Gambino and M. Misiak, Nucl. Phys. B 611, 338 (2001) [arXiv:hep-ph/0104034].

[4] P. Niezurawski, A. F. Zarnecki and M. Krawczyk, arXiv:hep-ph/0507006.

[5] P. Niezurawski, A. F. Zarnecki and M. Krawczyk, JHEP 0502, 041 (2005) [arXiv:hep-ph/0403138], Acta Phys. Polon. B 36, 833 (2005) [arXiv:hep-ph/0410291], LC-PHSM-2004-035

[6] G. Weiglein et al. [LHC/LC Study Group], arXiv:hep-ph/0410364. 